Mots. Les langages du politique

$104 \mid 2014$

Les livres de journalistes politiques

\title{
La contribution des New Journalisms au renouvellement du reportage politique aux États- Unis
}

The contribution of the New Journalisms to the evolution of political campaign reporting in the United States

\section{Erik Neveu}

\section{OpenEdition \\ Journals}

Édition électronique

URL : https://journals.openedition.org/mots/21568

DOI : $10.4000 /$ mots. 21568

ISSN : 1960-6001

Éditeur

ENS Éditions

Édition imprimée

Date de publication : 19 mai 2014

Pagination : 19-39

ISBN : 978-2-84788-542-2

ISSN : 0243-6450

\section{Référence électronique}

Erik Neveu, «La contribution des New Journalisms au renouvellement du reportage politique aux ÉtatsUnis », Mots. Les langages du politique [En ligne], 104 | 2014, mis en ligne le 19 mai 2016, consulté le 23 avril 2022. URL : http://journals.openedition.org/mots/21568; DOI : https://doi.org/10.4000/mots. 21568 


\section{La contribution des New Journalisms au renouvellement du reportage politique aux États-Unis}

Cette contribution ne se fixe pas la tâche démesurée de rendre compte des livres produits par les journalistes politiques aux États-Unis depuis un demisiècle. L'entreprise serait déraisonnable, tant au vu du flux de production que de la difficulté fréquente à fixer une identité professionnelle claire des auteurs, tant fluctue la frontier entre journalistes, agents opérant dans un espace flou de métiers de la production culturelle et - depuis les années quatre-vingt - idéologues conservateurs investissant l'espace médiatique. Il suffisait, au printemps 2013, de taper sur le site d'un grand marchand de livres en ligne le nom «Barack Obama» pour voir s'afficher plusieurs centaines de titres, dont une large part relevait d'une littérature de dénonciation dont la violence transforme en bluettes les pamphlets anti-Sarkozy ou anti-Hollande ${ }^{1}$.

L'objectif plus précis de ces pages serait de rendre compte du renouvellement apporté à la pratique du reportage politique, au premier chef au compte rendu des campagnes électorales, par ce qu'il est convenu d'appeler le New Journalism. Comme tout label, celui-ci fait l'objet de luttes en appropriation, dévalorisation ou redéfinitions. L'acte constitutif de ce journalisme est le manifeste éponyme que signe Tom Wolfe (1973) en tête d'un volume qui regroupe un florilège des textes qu'il associe à cette démarche, où il enrôle Didion, Herr, Talese, Thompson mais aussi Capote et Mailer. Le programme du New Journalism peut être condensé autour de la revendication d'une pratique de l'enquête en profondeur, de l'immersion dans l'univers à couvrir, avec souvent le souci de s'intéresser sinon aux gens « ordinaires », du moins à d'autres qu'aux élites, avec une attention particulière à ce qui manifeste le changement des styles de vie, des cultures, des valeurs dans les États-Unis des années 1965-1975. Le New Journalism, c'est aussi la revendication d'emprunts aux techniques du roman et de la littérature, avec des descriptions approfondies, une écriture indicielle,

1. Pour ne donner que deux échantillons aux titres évocateurs : Freddoso (2011) et Cullen (2012). 
une valorisation des dialogues et des procédés comme le monologue intérieur - le stream of consciousness à la Joyce - permettant de restituer ce qui advient - le mot est de Wolfe - «dans la tête des gens». Curieusement, ce New Journalism et ses avatars, qui ont significativement marqué tant l'écriture que les modes d'enquête journalistique aux États-Unis, font l'objet d'une asymétrie analytique. Il n'y a pratiquement aucune approche de sciences sociales sur cet objet, fortement investi par contre dans les années 1975-1985 par les narratologues et théoriciens des genres littéraires. Leurs contributions (Zavarzadeh, 1976 ; Hollowell, 1977 ; Hellmann, 1981) riches et parfois dissonantes se prêtent mal à un résumé simple. On peut en suggérer deux dimensions. Beaucoup de textes associent l'émergence de ces journalismes à ce qu'on pourrait appeler la dérobade du réel. Comment parler d'un monde où la réalité déborde la fiction quand l'homme marche sur la lune, quand on massacre dans les rizières sur fond sonore de Voodo Chile, quand un président des États-Unis espionne le parti rival et que la crudité de son parler dans le bureau ovale vaut celle d'un charretier? Comment maintenir le cadre épistémologique d'un observateur rapportant une réalité extérieure quand cette «réalité » peut être faite de media-events fabriqués pour les journalistes dont les comptes rendus auront à leur tour des effets sur le réel ? Et comment un journalisme objectivant rendil compte d'un monde peuplé de hippies, de pranksters dont le dessein est d'explorer des états psychiques qui échappent à l'observateur, de substituer au réel des voyages intérieurs (Wolfe, 1968) ? Peut-on d'ailleurs faire sens d'un monde qui semble échapper peu à peu aux prises analytiques des sciences sociales, à la simple idée de causalité, où la mosaïque des communautés fait vaciller l'idée de faire société, de partage des mêmes outils interprétatifs ? Sur un volet d'analyse discursive, ces textes questionnent la prétention centrale du New Journalism à combiner à la fois la «factualité immaculée » revendiquée par Capote dans De sang-froid et les ressources du roman. Mots valises (fictual) ou quasi-oxymores «non-fiction narratives » tentent de cerner ces régimes de mise en récit, questionnent la possibilité même de rapporter des faits réels sans oublis, montages ou condensations en une scène de data collectés de façon éparse. Ils questionnent tensions et coûts d'une combinaison du meilleur des deux mondes romanesque et journalistique.

La dimension littéraire du New Journalism ne tient pas qu'à ses emprunts revendiqués à la tradition du roman réaliste. Le courant manifeste une affinité naturelle avec le livre, que les reportages en prennent directement la forme ou que les livres naissent d'un travail de fusion et de recomposition d'une série de papiers qui faisaient chacun dix, vingt, trente pages. Le livre de journaliste est auxÉtats-Unis un objet dont la logique est singulière. Elle passe peu par la veine essayiste, typiquement française, bien davantage par de vrais reportages. La clé de cette formule tient à la place dans le paysage de la presse d'une série de magazines (Harper's, Esquire, Rolling Stone, Vanity Fair, The Nation, New 
York Review of Books) ${ }^{2}$ dont le récent $X X I$ donnerait un homologue français, où se publient des articles qui font banalement le format d'une «nouvelle» littéraire. La logique de ces magazines permet des durées d'investigation prolongées, qui s'étalent sur des mois, parfois des années. Si on y ajoute - pour une élite restreinte de reporters - des systèmes de financement par des fondations et des tirages considérables pour les best-sellers (qui se doublent d'un circuit de conférences, des droits liés à des adaptations cinéma ou documentaires), on entrevoit une «formule économique »3 qui aboutit à la sortie, chaque mois, de nombreux et souvent excellents livres-reportages de non-fiction narrative.

Ce New Journalism s'épanouit dans la période 1965-1975, donnant lieu à ce que l'écoulement du temps permet d'indexer aujourd'hui comme des chefsd'œuvre panthéonisés (Wolfe, 1965 et 1970; Thompson, 1966; Didion, 1968; Herr, 1977). Cette manière de pratiquer le journalisme a pu sembler souffrir d'une éclipse, voire d'un discrédit dans les années quatre-vingt. Avec moins d'arrogance à faire la leçon à la communauté professionnelle, moins de pyrotechnies rhétoriques à la Wolfe ou de forfanterie en style « couillu » à la Thompson, cette veine n'en est pas moins d'une notable vitalité dans la presse états-unienne d'aujourd'hui, avec des reportages issus de séquences exceptionnellement approfondies d'immersion et de suivi des sujets, qui s'attachent désormais aux classes populaires, aux mondes du travail, aux relations entre communautés ethniques (Harr, 1995; LeBlanc, 2003; Junger, 2010). Tout en poursuivant une logique d'emprunt aux techniques et formes proprement littéraires, cette nouvelle génération de journalistes mobilise aussi, plus que la précédente, des savoir-faire et des outils d'exploration du terrain qui viennent des sciences sociales (Neveu, 2012b), singulièrement d'une tradition ethnographique valorisée par les générations successives de l'École de Chicago, de Park à Becker. Robert Boynton (2005), professeur de journalisme à la New York University, a pu parler d'un New New Journalism dans un volume qui présente quelques contributeurs actuels à cette veine. Peut-être donnerait-on une idée de l'importance de ce courant en suggérant qu'alors que la presse et la critique française considèrent des reportages comme Le quai d'Ouistreham, de Florence Aubenas, ou Une saison de Machettes, de Jean Hatzfeld, comme des événements et des achèvements singuliers, il paraît aux États-Unis plusieurs livres de cette facture chaque mois, issus des reportages publiés par les magazines spécialisés.

Ce cadrage sommaire le laissera suspecter : les New Journalists ne sont pas des journalistes politiques. Leurvalorisation des soft news, leur attrait pour les profondeurs du social les vaccine plutôt contre toute fascination pour le jeu

2. Pour ne citer que des revues où publient les journalistes étudiés ici. Il faudrait ajouter à la liste des titres aussi centraux que le New Yorker, Atlantic Monthly.

3. Bien coté puisque déjà récompensé d’un Pulitzer en 1979, Ben Cramer recevra 500000 dollars d'à-valoir sur What it takes. Le chiffre, considérable, n'est en rien représentatif d'une moyenne. Il suggère les coups jouables sur ce marché. 
politique, sa centralité dans l'actualité chaude. Leur rapport à ce monde est oblique et épisodique, presque forcé. Ayant lu tant par plaisir que logique de recherche une petite centaine de volumes de reportages de ce courant sur la période 1965-2010, nous n'en avons pas trouvé dix sur la politique professionnelle et électorale4. Il fallut une sollicitation de la New York Review of Books pour que Joan Didion se mette - à près de cinquante ans - à écrire sur le sujet. Mais ces intermittents, ces «traîne-les-pieds » du reportage politique sont aussi ceux et celles qui, au vu de critères objectifs comme les rééditions répétées, les prix Pulitzer, les évaluations par les pairs et professionnels du journalisme, vont marquer l'art et la manière de couvrir une campagne électorale.

Si elle n'est pas inattentive aux dispositifs rhétoriques, l'approche proposée ici ne se revendique pas d'abord des sciences du langage. Elle cherche à identifier le «vraisemblable» dans un genre, celui du livre politique de journaliste, plus encore à comprendre les conditions sociales de possibilité de sa subversion. Le parcours proposé se structure en quatre moments, chacun polarisé par des auteurs et des reportages. Pour rendre compte du paradoxe apparent d'un renouveau apporté par des outsiders, il faut partir de ce qu'était, dans les années soixante, la manière normale - au sens kuhnien - de rendre compte d'une campagne présidentielle, celle des best-sellers produits de 1961 à 1973 par Theodore White dans sa série des Making of the President. C'est à partir de là que peuvent s'expliciter deux ruptures presque exclusivement portées par le New Journalism. La première, illustrée par Timothy Crouse et Joe McGinniss, ne concerne rien de moins que la définition des protagonistes sur lesquels il convient de se fixer pour comprendre une présidentielle. La seconde tient en une rupture radicale de ton : un ensemble de reportages ou de chroniques allant des années soixante-dix aux années quatre-vingt-dix va congédier le rapport de déférence aux acteurs, rites et institutions de la politique, exprimer la vision désabusée d'une activité perçue comme ayant oublié que son sens était de représenter des citoyens, de chercher les solutions à leurs problèmes. Un arrêt final sur le monstrueux livre de Richard Ben Cramer dédié à l'élection de 1988 suggérera combien un des effets, peut-être définitifs, du New Journalism en matière de reportage politique tient à ce qu'il soit devenu périlleux de raconter le combat politique sur le registre désormais suranné qui fut celui de White.

\section{Theodore White : une entreprise narrative de célébration (1961-1973)}

Journaliste initialement spécialisé sur la Chine, Theodore White va largement inventer un genre journalistico-littéraire : la chronique de campagne présiden-

4. Je remercie Rod Benson et Dan Hallin pour leurs utiles suggestions de lectures. 
tielle. Il l'inaugure en 1961 avec une longue et épique narration du duel Kennedy-Nixon : ce Making of the President 1960 sera un best-seller, prix Pulitzer 1962 en non fiction writing. Il inaugure une série de trois volumes ultérieurs en 1965, 1969 et 1973, mais aussi la floraison croissante de titres qui s'inspirent de la formule et du marché ainsi créés. La recette peut se condenser en quatre éléments. Le premier est un style narratif qui est celui de la quête, d'une épopée moderne. Elle met en scène des personnages portraiturés avec soin, même si l'investigation psychologique ou l'objectivation sociologique restent modestes. La structure des livres est fondamentalement chronologique, partant des primaires pour se resserrer sur le duel et se clore sur le moment du vote et du résultat. La dimension épique tient à la logique agonistique puisque les volumes sont aussi structurés par l'élimination ou les abandons des protagonistes initiaux au sein de chaque parti. Elle vient aussi, on y reviendra, de ce que pour White, aucune fonction au monde ne peut excéder l'importance de celle de l'occupant du bureau ovale et qu'il ne doute jamais des qualités hors du commun de ses héros. La dédicace aux frères Kennedy assassinés qui ouvre le Making of 1969 cite la Bible : «Plus vifs que des aigles, plus braves que des lions étaient-ils...»

Le mode d'écriture de White peut être qualifié d'objectivant. II porte un grand soin à éclairer les conditions de la campagne de données factuelles et chiffres qu'il peut emprunter aux recensements, à des sources statistiques (sur la diffusion des récepteurs TV par exemple) ou à des analyses de sociologie électorale. Le souci de fiabilité factuelle est évident dans la grande précision des dates, des localisations, des citations. La référence au « je» n'est jamais occasion de se mettre en scène, mais moyen de faire état de rencontres, de conversations directes avec les protagonistes, d'une proximité qui permet un dîner partagé à l'impromptu avec les Nixon un soir de primaire.

Un troisième trait de ces récits est leur ton déférent, faiblement critique. White est triplement persuadé de l'excellence des institutions politiques, de la qualité du personnel politique et de la suprématie de l'American way of life. Le préambule du volume de 1969 suggère bien les petitesses de la vie politique :

Les petits-déjeuners matinaux, la graisse de hot-dog et la moutarde sur leurs mentons dans les foires de comté, le serrage de mains à la chaîne aux portes des usines, les mornes collecteurs de fond, les hypocrisies obligées de présentation, les solutions minute, les arrangements sournois et fuyants - tout cela en 68 comme toujours dégrade les meilleurs des hommes. (1969, p.x)

Mais rien là que de bénin, au regard des qualités du système politique des États-Unis : «Dans un monde d'orthodoxies politiques rigides, d'États crispés par le dogme, l'Amérique, dans toute sa confusion, offrait encore des choix, elle saisissait encore partout les hommes à la pensée qu'on puisse trouver en tâtonnant un chemin vers des solutions. » Quant aux dirigeants politiques 
qui «prennent soin de ce petit vaisseau spatial5, qui tentent de l'organiser et de maîtriser sa course, [ils] demandent de la compréhension, et le dessein de comprendre conduit inévitablement au respect et à la sympathie» (ibid. p. x). Si White manifeste une admiration particulière pour les Kennedy - dont le père fut son condisciple à Harvard -, sa bienveillance est également distribuée dans les portraits emphatiques de Humphrey et Nixon, deux dirigeants issus d'un monde populaire de petites bourgades, dans l'évocation de l'activisme législatif du président Johnson (1969, p. 101). Les seules notations critiques ciblent ceux et celles qui commencent, spécialement en 1968, à défier le consensus américain : crazies du mouvement étudiant SDS dont «les glandes ont pris le contrôle du cerveau» (1969, p. 220, 286) et qui déclenchent la violence à la Convention démocrate de Chicago, Noirs dont les violences de rue, au lendemain de l'assassinat de Martin Luther King, ne semblent pas rationnellement explicables (1969, p. 209), et même une Cour suprême plus émue par la lecture de la Bible à l'école que par celle de la pornographie partout (1969, p. 34). Dans un compte rendu parallèle des livres de White et de Thompson sur la campagne 1972, où il donne pourtant une supériorité écrasante au premier nommé, Wayne Booth (1973), spécialiste de théorie littéraire, parlera d’un ton «mandarinal, un ton délibérément solennel qui implique victoires et défaites héroïques », flirte avec «la platitude, la composité et la solennité [...] White se laisse aller si souvent dans la fausse épopée. Vous ne pourriez pas deviner, en lisant White, que quoi que ce soit de drôle ait pu arriver à un candidat ou un reporter. »

Si l'on se fixe enfin sur ce qu'on peut nommer les cadrages, ils sont pour l'essentiel déterminés par le souci de rendre compte d'une compétition, donc par les tactiques, les échanges de coups, ce qu'on nomme la horse race politics. Vieille et visible figure du journalisme, White a les ressources d'un insider. Familier des acteurs du jeu politique, communiant à leurs catégories d'entendement, il acquiert cette proximité sociale et épistémique qui le rend d'une grande sagacité pour déchiffrer des coups politiques, des finesses tactiques. Il peut ainsi expliquer en quoi la candidature de Humphrey aux primaires de Virginie de l'Ouest est une bévue (1961, p. 96-97)... Une défaite y serait catastrophique, une victoire sans enjeu, tandis que les négliger eût rendu sans signification même un triomphe de Kennedy. Les analyses sur l'ambiguïté de l'entreprise nixonienne visant à courtiser à la fois le vote noir des villes du Nord et le socle de votes conservateurs blancs du Solid South sont encore éclairantes (1961, p. 315). Si sa narration est souvent explicative - d'autant qu'elle bénéficie du confort de synthétiser après la bataille - sur les dynamiques de campagne et les tactiques, cette valorisation a pour corollaire la moindre couverture d'autres dimensions. Les choix de fond, les politiques publiques n'intéressent White que pour autant qu'elles participent de la dynamique des échanges de

5. White reprend ici une métaphore d'Adlai Stevenson pour désigner la planète. 
coups, bien moins pour leur signification politique, les changements dont elles peuvent être porteuses. Mais son récit peut aussi faire penser - involontairement? - que ce rapport instrumental aux idées et programmes est celui d'une partie des candidats.

De façon rétrospectivement clivante, c'est aussi le traitement de ceux qu'on pourrait nommer les adjuvants du combat électoral qui vaut d'être relevé. White ne néglige ni les journalistes, ni les organisateurs, mais il questionne modestement la nature de leur rôle. Il peut être sensible à la qualité des personnes et de l'organisation qui entoure un candidat. Il loue la sûreté de Robert Kennedy à bien s'entourer, admire la machinerie bien huilée de la grosse équipe Kennedy de 1960 (1961, p. 323), s'amuse de l'efficacité imprévue de l'armée d'étudiants d'Ivy League qui vient au secours de McCarthy en 1968. Mais la perception est fonctionnaliste. La machine tourne-t-elle bien, est-elle réactive, couacs et désynchronisations sont-ils conjurés? Si White est très sensible au pouvoir des vieux bosses et financeurs comme Daley ou Rockfeller, la possible ascension des consultants et communicateurs dans les campagnes le questionne peu. Une observation parallèle vaut pour les journalistes et les médias. White n'y est nullement inattentif. Le volume de 1961 consacre un plein chapitre aux quatre débats télévisés qui constituent alors une innovation, en tirant le bilan sans mythologiser le fameux épisode de la barbe noire de Nixon qui lui aurait donné une physionomie patibulaire (Neveu, 2012a). Mais si White souligne la présence constante, parfois envahissante des journalistes et montre - en comparant explicitement l'attitude du Nixon, de 1960 et de celui de 1968 (1969, p. 143, 327) - qu'il n'est pas indifférent de se ménager leur bienveillance, il ne théorise guère la signification et a fortiori l'ambivalence de la relation symbiotique journalistes-candidats. II a, certes, l'œil sur l'ordinaire de leurs interactions :

Des semaines et des mois durant ils doivent vivre comme des vagabonds - secoués, bousculés, se colletant avec les cordons de policiers, sales et sans avoir pris de bains des jours durant à la fin, cornaqués dans des bus comme des écoliers. L'attention aux correspondants, le soin pris d'eux était très similaire dans ces deux états majors mobiles - mêmes communications instantanées par téléphone ou télégraphe, même plateaux-repas, mêmes arrangements avec les hôtels pour les bagages, les repas, la blanchisserie. Mais rien dans cette logistique méticuleuse d'accompagnement ne pouvait gommer la turbulente réalité du reportage, la folle qualité des vols en jet de la campagne 1960, ni protéger la simple dignité individuelle d'hommes qui devaient combiner les qualités de philosophes et de portefaix dans des circonstances qui les dépouillaient inexorablement de leurs dernières parcelles de dignité. (1961, p. 335)

L'observation ethnographique est juste. Mais Crouse permettra de voir qu'elle passe à côté de l'essentiel : les enjeux sont autres que d'hygiène ou d'élégance. Il faut d'autres concepts que dignité pour comprendre les interdépendances qui se créent. 


\title{
Redistribuer les rôles politiques : McGinniss, Crouse (1969-1973)
}

\author{
Faites entrer les consultants...
}

Les limites du potentiel critique des narrations de campagne par White deviennent bien visibles avec les reportages produits dès la fin des années soixante. Venu du journalisme sportif, columnist au Philadelphia Inquirer, Joe McGinniss accède, à 26 ans, à une fulgurante célébrité avec un livre sur la campagne Nixon de 1968, dont le titre est en soi une prise de distance à la déférence whitienne: The Selling of the President, 1968 (1969). Le livre sera six mois durant dans la liste des best-sellers. Le parti pris de McGinniss - né d'une conversation où un collaborateur de Humphrey lui annonce que son employeur va retourner l'opinion par une communication pensée stratégiquement - consiste à se fixer sur le rôle des communicants et stratèges en marketing politique pour les considérer non comme de simples auxiliaires du combat politique, mais comme des acteurs centraux. McGinniss fait d'autant plus mouche qu'après avoir échoué à accéder au cœur de la machine de campagne d'Humphrey, il voit, d'une manière rétrospectivement surprenante, l'équipe Nixon lui ouvrir généreusement accès aux archives et aux stratèges de campagne.

Le reportage met d'abord en lumière le cynisme des communicateurs, totalement étrangers à la vision enchantée d'un citoyen rationnel cherchant à s'informer des enjeux pour choisir en comparant engagements et programmes. Les «notes » de divers conseillers valent d'être citées. Ainsi l'un affirme-t-il :

Les électeurs sont fondamentalement paresseux, fondamentalement peu motivés à faire un effort pour comprendre ce dont nous parlons... La raison requiert un haut degré de concentration et de discipline, l'impression est plus confortable. La raison repousse le spectateur, l'agresse, lui demande d'approuver ou de désapprouver. L'impression peut l'envelopper, l'inviter sans faire de demande intellectuelle. [...] Quand nous argumentons pour lui, nous lui demandons de faire l'effort de répondre. Nous tentons de mobiliser son intellect et pour beaucoup de gens c'est la plus difficile des tâches. Les émotions sont plus facilement atteintes, plus proches de la surface, plus malléables. (p.33)6

Un autre conseiller met en garde :

La politique n'est que bien peu une science rationnelle. Les arguments ne seront efficaces que si nous pouvons amener le public à faire un saut émotionnel ou ce que les théologiens nomment le saut de la foi [...] Nous devons être très clairs sur ce point : les réactions se font sur l'image, pas sur l'homme. (p.172 et 174)

6. Ma traduction, ici comme pour toutes les citations d'ouvrages non édités en français. 
McGinniss montre comment la campagne est à la fois un théâtre d'ombres, une série ininterrompue d'événements pour médias et de performances calibrées pour toucher des cibles précises, accrocher le sens commun ou des émotions. L'ouvrage illustre le double professionnalisme des publicitaires en campagne et du personnel politique. L'une des premières scènes du livre montre Nixon enregistrant des spots publicitaires pour la télévision, évaluant à chaud, à la seconde près - sans horloge ni montre - la durée de son propos. L'ouvrage rend intelligible à quel point, dès 1968, la notion de campagne télévisée flirte avec le pléonasme : "Nixon dépendait du studio télé comme un poliomyélitique dépend du poumon d'acier» (p.123). Toute la logique de Nixon consiste à limiter au maximum les interactions avec une presse écrite tenue pour inamicale, pour y substituer la mise en scène de situations maîtrisables (comme des panels de débat aux participants choisis), de messages calibrés pour les studios de télévision, à focaliser les attaques sur la personne ou le patriotisme de leur adversaire plus que sur les programmes. La campagne peut bien, selon McGinniss, «porter la platitude à la hauteur d'un art» (p.104), ces platitudes mobilisent le sens commun, les valeurs gratifiantes de l'américanité... les électeurs seraient comme des enfants qui, le matin de Noël, prêtent moins attention au jouet qui leur est offert qu'à la boîte et au papier qui l'enveloppent (p. 26).

Le succès du livre de McGinniss doit au moins à deux ressorts. Il est le premier à axer le projecteur sur les communicateurs et marketeurs politiques, en l'occurrence autant piégés que manipulateurs. Parce que nourri de documents bruts, de multiples «choses vues » lors de mois de participation à la campagne, il a la force d'un témoignage peu contestable. Le fait que la démonstration porte sur un dirigeant qui ne fut jamais tenu pour un parangon de vertu la sert probablement. On se demandera cependant si elle ne comporte pas une part de naïveté. Le langage des publicitaires est cynique. Mais est-ce son unique sens? $\mathrm{Ne}$ reflète-t-il pas une forme d'intelligence pratique de ce que rendent sociologiquement intelligibles des travaux comme ceux de Daniel Gaxie (1993)? Prendre acte d'un faible intérêt pour la politique d'une majorité des électeurs, et par-là de leur recours à des raccourcis cognitifs, des logiques d'allodoxia et de remise de soi, est-ce un crime de lèse-démocratie, ou la conscience de ce que celleci repose sur un cens caché que peu de ses hérauts s'emploient à réduire?

\section{La force d'un regard spéculaire}

The Boys in the Bus, le reportage de Timothy Crouse (1973) portant, lui, sur la campagne de 1972, va conforter ce processus de recomposition, de repeuplement au sens latourien, des acteurs d'une campagne électorale. Journaliste au magazine rock Rolling Stone, Crouse est envoyé par sa rédaction - en même temps que son collègue Hunter Thompson - couvrir la campagne qui aboutira au duel Nixon-McGovern. Le coup de génie et l'œuf de Colomb de Crouse sont 
de ne pas considérer les journalistes comme les chroniqueurs plus ou moins fidèles d'une campagne dont ils sont les témoins... mais de les penser comme des acteurs à part entière du processus électoral, des associés-rivaux pris dans une relation ambivalente aux candidats, ne serait-ce que parce que les préséances dans les rédactions sont liées au poids, au parcours du candidat que chaque reporter suit : «Il ne fallut pas beaucoup de jours à être véhiculé dans ce bus pour voir que les reporters eux-mêmes feraient un fort bon sujet.»

Le reportage de Crouse confirme en tous points celui de McGinniss quatre ans plus tôt quant à la pérennité d'une animosité de Nixon pour les journalistes de presse écrite, à l'expertise de son équipe à construire une campagne largement circonscrite à une grosse bulle d'enregistrements en studio, de media events bien contrôlés, de bains de foule conjurant au maximum le risque d'imprévu. Son livre va aussi donner naissance à l'expression devenue banale de pack journalism, journalisme de meute. Là où White discerne la promiscuité durable entre candidats en campagne et journalistes mais en limite l'analyse aux déplaisirs d'une vie de chambrée transportée dans de bons hôtels et des jets, Crouse questionne des rapports de pouvoir. Au sein du pack, les "poids lourds», journalistes consacrés ou appartenant à des titres prestigieux, tiennent en quelque sorte le diapason des sujets et tonalités de couverture. Les effectifs suffisent parfois à comprendre le sens de "poids lourd» quand Time envoie cent trente personnes à la convention démocrate de Miami. Ce premier effet de clôture est redoublé par le huis clos d'une troupe où l'interconnaissance est intense (les scènes d'effusions, les yeux humides lors de la séparation finale sont parlants...) et où les journalistes, quels que soient leurs talents, sont portés aux mêmes questions tant par l'habileté des communicants qui s'emploient à orchestrer l'agenda que par le simple fait de ruminer les mêmes communiqués, discours et pseudo-événements avec des catégories de pensée largement partagées. La clôture, c'est encore celle qui consiste à osciller entre la vacuité sociale d'une «vision du monde à trente mille pieds » dans les avions qui véhiculent la troupe et celle des spectacles mis en scène par l'équipe de campagne, attentive à faire des boys in the bus des spectateurs et non des trublions qui poseraient des questions agressives. Crouse montre encore comment ce fonctionnement en chorale, faiblement capable de distanciation critique, est amplifié par la norme d'objectivité qui impose de rendre compte des propos d'un candidat - même si on les tient pour démagogiques ou mensongers. Elle rend difficile l'acceptation par les rédactions de reportages à tonalité interprétative. La crainte être accusés de partialité fait aussi que les journalistes du pack, plus souvent liberals que conservateurs, sont en fait plus sévères avec McGovern qu'avec Nixon.

Crouse ne décrit en rien un pack uniment fait de victimes consentantes. Il suggère comment des techniques d'objectivation - le precision journalism (Meyer, 1973) - comme le recours à des données de sociologie électorale, 
la production d'enquêtes originales sur les attentes des électeurs pourraient réarmer les journalistes. II montre aussi que le groupe des poids lourds a pu prendre depuis les années soixante un pouvoir d'adouber et de coter les candidats sérieux aux primaires, parfois avec deux ans d'avance, qui concurrence les machines de parti. Mais il souligne plus encore le poids stérilisant des logiques combinées des publicitaires, des candidats et des routines professionnelles. Il relève la faible capacité du groupe professionnel à agir collectivement, à se rebeller face aux humiliations ordinaires d'une équipe de campagne qui les cornaque et les tient à l'écart du président-candidat, à oser appuyer les plus audacieux, comme Woodward et Bernstein du Washington Post, initialement fort solitaires dans leur investigation du Watergate.

On peut suggérer quelques points communs aux reportages évoqués ici. Ils sont le fait de non-spécialistes du journalisme politique. Ils sont aussi issus d'une présence intense et prolongée sur le terrain, mais d'une présence qui n'est pas celle d'insiders mais de journalistes encore capables de se demander ce qui est important, ce qu'il faut regarder dans une campagne politique. Ces reportages s'expriment dans des textes longs, soit directement en livre, soit par de gros articles dans Rolling Stone. Enfin, sans faire de ces journalistes des Monsieur Jourdain de la sociologie politique, leurs observations sont aisément appropriables dans des catégories de la pensée sociologique (chaînes d'interdépendance, routines professionnelles, effets d'allodoxia)... Le livre de Crouse demeure d'ailleurs cité dans nombre de travaux de sociologie du journalisme.

\section{Du plaisir d'insolence au devoir d'irrespect : Thompson, Didion, Lewis (1973-2000)}

\section{Un reportage sarcastique et combatif}

Crouse avait, sur la campagne 1972, sinon un équipier, du moins un collègue de rédaction: Hunter Thompson. Rendu célèbre par son reportage de 1966 sur les Hell's Angels, Thompson est lui aussi lancé sur la piste de la présidentielle par Rolling Stone. Il y développe les méthodes d'observation intensive du New Journalism en y ajoutant sa marque de fabrique : le gonzo journalism. La posture consiste ici à ne pas suivre la méthode Wolfe qui est d'être «la mouche sur le mur», observant sans être vue, mais d'être «la mouche dans le verre de lait» : créer par des questions inconfortables, un comportement agressif ou imprévisible (Thompson consomme beaucoup de produits qui agissent sur le psychisme), le malaise, la situation révélatrice. Nourrissant une aversion militante pour toute forme d'autorité, Thompson prend un malin plaisir à tourner en dérision les politiques. Il ne cache pas la haine que lui inspire Nixon. Ses articles engendrent un pavé (1973) de plus de 500 pages. Sa logique narrative 
n'est paradoxalement pas aux antipodes de celle de White. On y suit la campagne, de primaire en primaire, puis dans le duel final. Les péripéties tactiques, les ambiances dans les équipes de campagne priment sur l'analyse des propositions ou programmes. Le résultat final n'en est pas moins original.

Ce cachet tient au premier chef à un refus argumenté du journalisme objectif - dont Thompson soutient que l'illustration pure et soporifique serait les systèmes de vidéosurveillance des grands magasins. Il récuse aussi les jeux de dons et contre-dons policés :

En ce qui me concerne, il n'avait rien de tel que du off the record. L'échec le plus durable et en dernière analyse le plus dommageable du journalisme politique en Amérique prend ses racines dans les relations personnelles au parfum de club ou de cocktails qui se développent inévitablement entre politiques et journalistes [...] Quand des professionnels en antagonisme deviennent en fin de journée compagnons de bistrot, ils ne risquent pas de s'affronter bien sérieusement. (p. 18)

Le refus du verbe objectif donne un ton insolent, parfois insultant, autorise des séquences imaginaires assez faciles à déceler pour que la tromperie soit bénigne. L'un des candidats démocrates, Muskie, est une des têtes de Turc du récit : «Il y avait une odeur de mort autour de lui. Il parlait comme un paysan en phase terminale de cancer qui aurait tenté d'emprunter de l'argent sur la récolte de l'année suivante» (p.144). Et Thompson de lancer le bobard, bien entendu aussitôt débattu au sein du pack, selon lequel Muskie se drogue à l’ibogaïne, une mystérieuse plante brésilienne. Le parti pris « gonzo » est aussi de tenter de joueur un rôle dans la campagne, d'exprimer sans litotes son aversion pour Nixon, sa sympathie exaspérée pour un McGovern dont on comprend qu'il aurait dû prendre Thompson comme conseiller en communication. Ce que le livre suggère aussi - théoriser serait un verbe surdimensionné -, c'est une forme de dépit, de deuil de ne pouvoir trouver un dirigeant politique simplement décent, qui soit plus attentif aux citoyens qu'aux coups tactiques à jouer pour occuper le pouvoir, qui soit capable de sortir du microcosme de Washington, de l'entre-soi politicien. La sympathie de Thompson va clairement à McGovern, aux étudiants idéalistes qui se donnent sans compter pour sa campagne. Mais ce qui engendre «peur et dégoût» sur la piste présidentielle, c'est bien qu'un tel candidat, pourtant encore trop politicien, n'ait pas raisonnablement de chances de gagner. Plus souvent limités à de courts billets ou de petits reportages, les chroniques politiques (2010) de Thompson déplaceront ensuite sur Reagan ou les Bush l'aversion anti-nixonienne, sans jamais plus pouvoir nourrir grande illusion sur un démocrate?.

7. Thompson se suicide en 2005 , avant la candidature Obama, donc. 


\section{Les récits de la distanciation}

Le recueil des articles (2000) écrits à partir de 1988 par une des plus grandes plumes du New Journalism, Joan Didion, est un excellent révélateur. Admiratrice de Goldwater en 1964, critique face aux contre-cultures des années soixante, Didion n'a rien d'une radicale. Or tous ses reportages expriment une palette de réactions qui oscillent entre tristesse, dégoût et mépris quand elle parle de politique, d'une politique où elle pense les journalistes comme acteurs à part entière. La description qu'elle donne est celle d'un monde d'insiders devenu autiste à se passionner pour ses luttes, ses ruses tactiques et ses enjeux ésotériques. Les journalistes politiques en sont arrivés à saluer comme une performance l'art de ne pas répondre aux questions; un dirigeant de CBS peut s'indigner publiquement qu'un candidat réponde à un amphi d'étudiants en regardant les questionneurs et non les caméras. Le taux phénoménal d'abstentions, l'incapacité du Parti démocrate à prendre en compte les attentes de sa base sociale attestent de la profonde déconnexion entre l'univers politique et les citoyens. Didion déploie ses critiques sur deux fronts. Elle cible les schèmes d'analyse ressassés des journalistes, leur manière de penser la politique comme devant être un spectacle pour la télévision et non la construction d'un projet de vie collective avec des citoyens. Un de ses articles est une exécution de Bob Woodward, ex-héros du Watergate devenu un insider narcissique. Ravi de transcrire ses multiples conversations avec les puissants, Woodward ne se poserait plus la question du pourquoi de leur disponibilité, de leurs possibles stratégies face à un journaliste. II produit sur la campagne de Clinton 1992 une narration (1996) à la White, un livre «dans lequel toute trace d'activité cérébrale est virtuellement absente ». Didion clôt son papier en commentant la manière dont Woodward rapporte la visite qu'il fait, à son lit de mort, à un protagoniste clé d'un scandale, dont il espère aveu et regrets. «Dévoyer des moments si intimes sur le papier, c'est cela que veut dire mettre "la dimension humaine au cœur" du texte, c'est aussi cela que veut dire écrire de la pornographie politique. » Si Didion est dans la continuité de Crouse et McGinniss, elle exprime aussi une amertume, le désir déçu de pouvoir investir une illusio forte et sérieuse dans la politique comme moyen de changer le monde.

Représentant du New New Journalism célèbre pour ses reportages sur le monde de la finance ${ }^{8}$, Michael Lewis va lui aussi opérer à contre-emploi pour couvrir les primaires républicaines de 1996. La tonalité de son récit (1997) est proche de celle identifiée chez Didion. Croquis et épisodes restituent une politique qui est un art de fuir les problèmes, où les compétiteurs finaux ne disent

8. Dont le livre inaugural Liar's Poker (1990) raconte son expérience dans la banque d'affaires Solomon Brothers. 
rien de saillants sur les big problems de la protection sociale, des inégalités, de la décomposition des centres urbains, du poids de l'argent sur les élections. Dole comme Clinton «ont déployé une habileté étonnante pour feindre un engagement à l'égard du monde qui les entourait alors qu'en fait ils se cachaient» (p.xı) :

Les deux hommes veulent beaucoup, vraiment beaucoup être président, c'est dire qu'aucun ne veut perdre pour une cause. Ils n'ont même pas de mauvaises intentions dans leur vacuité et je n'essaierai pas de l'invoquer contre eux. Ils veulent juste gagner, et dans ce climat, la vacuité est apparemment un avantage. (p. 131)

Lewis décrit lui aussi un univers clos, élitiste et régi par l'argent, loin des réalités des Américains modestes. Il n’y a plus, soutient-il, ni droite ni gauche, mais des professionnels de la politique qui «vont de haut en bas d'un mât de cocagne graisseux, les carrières sont plus importantes que le sort de la planète» (p.18). Lors de la convention démocrate, il décrit une scène où des femmes de ménage misérablement payées - et dont les démocrates visent le vote - doivent jeter des milliers de dollars d'aliments raffinés parce que l'équipe du président sortant n'a pas jugé bon d'annoncer son absence à une soirée de gala (p.219). Moins critique que Didion pour les journalistes politiques, il observe que si leur prose semble «loin de la vie, c'est que la politique s'est déconnectée de la vie réelle» (p. 263) et que l'apathie politique apparaît alors comme une réponse plus logique qu'inexplicable. Pas plus que Thompson ou Didion, Lewis ne cotise au mythe de l'objectivité. Il indique au lecteur que son vote a été à Ralph Nader... ce qui donne une signification singulière à une série de jugements qu'il porte au fil des pages. Son compte rendu laisse en effet filtrer du respect ou de l'admiration pour quelques-uns des candidats les plus conservateurs (Buchanan, Keyes, Taylor) qui ont la singularité de mettre dans leur campagne autre chose que du métier et de la tactique : des croyances sincères, l'audace de porter des points de vue qui fassent autre chose que cibler l'électeur médian, des formes diverses d'intérêt vrai et d'affection pour les gens ordinaires. Il décrit ainsi le style de campagne de Buchanan comme «ouvert, honnête, amical et bienveillant» (p. 92). Buchanan parle une langue intelligible, pas un code. À l'exact inverse de Dole, ses réunions attirent les gens, mais pas les journalistes... dont tout le livre montre aussi la propension à voler au secours de la victoire, à coopter d'entrée les candidats «sérieux» dignes de leur attention et de leurs productions.

Le double exercice de reporters et d'évaluateurs de la vie politique auquel se livrent les représentants du New Journalism est convergent. On peut s'intéresser à une campagne comme à un match ou une compétition avec ses rebonds, ses fourberies, ses batailles, mais la politique s'est en quelque sorte retirée du réel. Elle ne fait plus sens pour la masse des Américains, ni comme écho à leurs soucis, ni comme une activité qui puisse significativement amé- 
liorer leur existence. Elle a conforté sa dimension d'activité de professionnels plus polarisés sur l'entre-soi et leur (ré)élection que sur le défi des problèmes publics. Elle est de plus en plus tributaire de niveaux considérables de financements non publics. Si le New Journalism condense cette approche critique, il n'en est pas une expression marginale. Pour un ensemble de raisons qui doivent à la fois au moment Watergate, au malaise né d'un sentiment d'instrumentalisation par les communicateurs, le journalisme politique américain a développé depuis les années soixante-dix une lecture plus défiante, plus critique de l'action des présidents (Clayman et al., 2010). On trouvera aussi sous la plume de journalistes d'investigation', comme de journalistes politiques appartenant à des titres centraux de la presse d'information politique, des critiques qui rejoignent celles explicitées ici ${ }^{10}$.

On doit alors se poser la question des raisons d'une humeur critique que le New Journalism concentre plus qu'il n'en a le monopole. Mécanisme de défense d'un groupe professionnel contre le trop d'habileté des politiques? Derniers soubresauts de l'humeur anti-institutionnelle de la génération des baby boomers ou de celle des quinquas? Ces facteurs opèrent. Ils sont seconds par rapport aux évolutions concrètes de l'activité politique, au double mouvement de clôture sur ses enjeux ésotériques d'un champ politique qui a intégré journalistes et consultants, et de dépendance accrue de ce champ à un flot de contributions financières privées aussi indispensables que génératrices de contredons contraints, de sujets tabous et de décisions impossibles. Leur extériorité au microcosme, leur statut transitoire dans le reportage politique rend le regard des journalistes évoqués ici plus critique, les protège de cet apprivoisement si bien rendu par la formule going native. La lecture que nous proposons est de souligner que ces récits ne relèvent ni de l'humeur, ni de l'humour, ni d'on ne sait quelle dénonciation «populiste $~{ }^{11}$ : Ils disent, sur la base d'une immersion aux côtés des acteurs - sans grands équivalents, y compris chez les chercheurs

9. Voir le livre controversé de Seymour Hersh (1997), présentant avec beaucoup de faits à l'appui la famille Kennedy comme débitrice envers la Maffia, peu regardante sur les méthodes du combat politique, et John Kennedy comme un prédateur sexuel pathologique.

10. Si son auteur anonyme - qui se révélera être Joe Klein, de Time - n’appartient pas au New Journalism, on doit citer le roman best-seller Primary Colors publié en 1996 et mis en film en 1998. Il raconte, avec un réalisme jugé saisissant par les journalistes politiques, la campagne de Jack Stanton, ambitieux gouverneur du Sud où chacun reconnaît Clinton, jusque et d'abord par les récits de frasques et gaffes sexuelles. Le récit avance d'un cran dans la narration réaliste d'une campagne, y intégrant les épisodes de sexe et les affaires entre activistes. Si Stanton apparaît comme un habile capable de rentrer dans de vrais échanges, voire des moments de communion avec des auditoires populaires, ayant des séquences d'authenticité émotionnelle, le roman suggère avant tout l'image d'un monde politique où convictions et programmes sont sans grande importance objective ou subjective, d'un monde où tous les coups sont permis : l'instrumentation des alliés et des aides est la norme, la capacité à encaisser les coups et à les rendre est la vertu cardinale du «métier». Klein publiera aussi en 1996 un livre sur ce qu’il nomme «le complexe industriel sondeurs-consultants ». Sur la clôture du microcosme politique, lire aussi Washington de Meg Greenberg (2001), du Washington Post.

11. Sur les raisons de ne pas utiliser ce concept spongiforme, voir Collovald (2004). 
en science politique - un état de la politique professionnelle, une crise du politique dont la clé ne vient pas de l'irréalisme des peuples devant la loi d'airain de l'austérité ou les exigences indiscutables de l'économie, mais de processus d'enkystement des activités politiques et d'appauvrissement de l'offre et des espaces d'intervention revendiqués par les acteurs politiques (Crouch, 2004 ; Hay, 2007).

\section{(Faux) épilogue. "What it Takes... " Ben Cramer et l'impossible retour d'un " Making of... "}

Donner sa place au singulier livre de Richard Ben Cramer (1992) sur la campagne de 1988 Bush-Dukakis était à la fois une nécessité et une gageure. Comment parler d'un livre de 1047 pages imprimées en corps 10, ayant demandé quatre ans de rédaction à partir d'un matériau documentaire colossal ? Il n'y a probablement aucun livre, fictionnel ou de reportage, académique ou journalistique qui restitue avec un tel luxe de détails, une telle profondeur de champ, une campagne électorale.

Dans sa conception, l’ouvrage est à bien des égards banal. S’il déploie une grande sophistication dans les retours en arrière, il donne à voir de façon chronologique le processus complet d'une campagne des élections de mi-mandat de 1990 à l'investiture de Bush en 1992. Mais une première singularité tient à l'angle de lecture retenu, explicité dès les premières pages : «Je voulais savoir non pas sur la campagne, mais sur les compétiteurs [...] Qu'est ce qui dans leur histoire peut leur donner cette immense émotion, cette sorte de moteur, cette volonté et cette discipline, la foi en eux-mêmes» (p.vII). Qu'est ce qui donne la conviction qu'on peut diriger la première puissance mondiale? Pour y répondre, Ben Cramer mobilise trois ressources. Une posture : l'empathie revendiquée pour tous les candidats. Un travail d'enquête stakhanoviste qui cumule la présence aux côtés des candidats, une collecte de mille entretiens avec l'aide de ses collaborateurs, une vérification systématique de tout propos ou déclaration rapportée dans le livre. La focale enfin est celle d'une grande profondeur; le livre contient en fragments des biographies de tous les acteurs majeurs, nous faisant remonter à l'éducation de Georges Bush qui en fera un compétiteur féroce, à la blessure sur le front italien qui, en 1943, laissera Dole handicapé à vie.

Ce parti pris permet de mettre en avant des dimensions peu présentes dans les reportages déjà mentionnés. La première est de déployer sur un temps long, avec un grand luxe de micro-interactions et de croquis sur le vif, ce qu'est le quotidien du «métier» politique. Tout politiste intéressé par le sujet devrait à ce titre lire le livre. On y saisit l'importance et la lenteur des processus de tissage de réseaux, de constructions de fidélités, la microéconomie du don et du 
contre-don... dont cette étonnante industrie de la relation personnalisée que sont les trente mille cartes de vœux que Bush expédie chaque nouvelle année. On y comprend aussi, grâce à l'attention au grain du quotidien, aux petits et grands drames personnels, la violence et le stress du combat politique. Cette tension est celle des défaites et des meetings ratés malgré de gros investissements, des coups bas des adversaires, parfois des proches quand Dole s'aperçoit qu'à son insu, son directeur de campagne a lancé contre Biden une attaque aussi ignoble que dévastatrice ou quand, humilié par Nixon, il réalise qu'il vient de remettre les clés du parti à Bush pour la ridicule gratification d'un sweat-shirt aux armes de la maison Blanche. Cette violence, c'est encore la destruction de Gary Hart, mis au pilori pour ses relations féminines alors même qu'il est en processus de divorce, c'est l'épouse d'un candidat qui gère la tension par un alcoolisme qu'elle dissimule, c'est encore l'agressivité d'une presse qui n'attaque pas sur les engagements mais sur un discours plagié, une maladresse de jeunesse occultée. La douleur de la politique ordinaire, c'est de ne pouvoir tenir des promesses réfléchies parce qu'un sortant peu vertueux a masqué l'énorme déficit que découvre le gouverneur fraîchement élu. Ce qu'apporte enfin le livre, c'est non La clé, mais des éléments pour comprendre les bêtes politiques que sont les candidats. Beaucoup ont subi des épreuves hors du commun : perte ou terrible maladie d'un enfant, blessure de guerre dévastatrice, bégaiement à conjurer, éducation familiale en forme de dressage. Ils se sont souvent faits dans un processus combiné de franchissement d'énormes défis personnels et d'apprentissage de sociabilités qui consistent à bâtir des réseaux, à se rendre utile, visible et sympathique. Ils ont aussi appris en donnant à leur rapport à la politique le tour d'une carrière, avec toutes les ficelles d'un métier qui est de passer à travers les gouttes, de conquérir une aisance de la parole faussement impromptue, un art de mobiliser des alliés et de neutraliser des adversaires où l'acteur lui-même ne démêle plus le jeu, le cynisme, le goût d'entreprendre ou de pousser des projets. Dans une formule sibylline, Weber dit du métier d'homme politique (1918) qu'il est «un effort tenace et énergique pour tarauder des planches de bois dur ». Ben Cramer réussit à rendre cet art parfaitement intelligible, mieux sans doute que maints travaux de science politique, parce que la dimension presque épique de son enquête, en nombre d'interlocuteurs, en amplitude temporelle, aboutit à collecter une masse prodigieuse de données, de petits faits parlants et que son talent de narrateur lui permet, dans un format proustien, de montrer en quoi le temps passé, le temps perdu du tissage de liens et d'obligations, engendre aussi ressources, réseaux et dispositions qui sont nécessaires aux plus hauts niveaux du champ politique.

On devine l'ambivalence du livre. Il a la structure de celui de White, mais en a désinvesti l'illusio du rêve américain, l'enchantement de la meilleure démocratie réellement existante. Il est, comme Crouse, Didion ou Lewis, dans une approche réaliste et froide des relations entre presse et candidats, dans une 
mise en évidence de la dureté, souvent de la clôture du jeu politique, mais il n'en dévalorise pas les acteurs. Chaque lecteur répartira sympathies et animosités selon sa subjectivité socialement bâtie, mais la narration ne met en scène ni monstres, ni pantins d'un système. Les hommes - bien rarement les femmes - qu'elle dépeint ne sont jamais sans qualités. Cette odyssée électorale rend intelligible la confluence d'histoires singulières et de pesanteurs systémiques qui font du métier politique ce qu'il est, qui sélectionnent les porteurs de certaines dispositions. À ce titre, Ben Cramer se sort fort bien d'une difficulté qui est aussi celle du travail sociologique. Comment objectiver avec rigueur sans écraser la singularité des cas et trajectoires (Neveu dans Von Heyking et Heilke, 2013)? Mais s'il restitue aux professionnels de la politique une individualité et souvent une humanité, il les met en scène prises dans un singulier jeu social. Ici les idées, les propositions réfléchies pour rendre la vie commune meilleure sont de peu de poids; l'argent détermine les rapports de force, le cynisme et les attaques ad hominem sont des armes décisives. La morale paradoxale de What it takes pourrait bien être qu'on peut s'intéresser aux joueurs, admirer leur technique et leur énergie, mais qu'il y a davantage lieu de leur prêter attention qu'au jeu et aux enjeux. Le réenchantement du politique n'est pas en vue.

Faux épilogue, pas seulement parce que le livre de Ben Cramer n'est pas le plus récent du corpus, mais parce qu'il faudrait bénéficier d'un double recul supplémentaire. L'un consisterait à disposer de bilans et récits argumentés de ce qu'auront été les années Obama, du prodigieux moment de mobilisation et d'espoir de la campagne de 2007-2008 aux frustrations des lendemains de victoire. Le second serait littéraire et consisterait à discerner ce que seront les livres sur ses campagnes ou son action qui survivront au temps. On disposera là d'un outil précieux pour savoir si des rémissions peuvent exister dans un processus de désenchantement dont notre corpus suggère les fondements structuraux.

Le journalisme politique connaît des formes plurielles (Kaciaf, 2013). Celui des spécialistes a ses points forts, singulièrement une intelligence des échanges de coups, des tactiques propres à l'art du combat politique. Il est aussi menacé des cécités issues du partage des catégories et des évidences des acteurs. II risque de ne parler qu'au cercle restreint des publics à la fois politisés et identifiés aux acteurs en situation de gagner. Les new journalists suggèrent à la fois les modalités d'une autre approche et ses conditions de possibilité. Parce qu'ils redoutent que se loge sous l'objectivité et la déférence une adhésion aux prétentions des institutions, une naturalisation de leurs normes, ils regardent la légitimation du politique comme service de la démocratie et du bien commun comme un objet à questionner et non un tropisme irrépressible des candidats. Ils élargissent la focale et intègrent aux protagonistes du combat poli- 
tique consultants, communicateurs, associés-rivaux du journalisme. Limitant leur contribution à des incursions momentanées, refusant l'engrenage du don contre-don, brûlant leurs vaisseaux par l'objectivation abrasive de leurs analyses ou l'agressivité de leurs comportements, ils font en sorte de ne pas devenir membres de la tribu. Qu'y perd-on? Une part de la pénétration des ruses des acteurs, la ronronnante assurance que la démocratie représentative répond à ses propres justifications. Qu'y gagne-t-on? Une forme d'intelligence sociologique des règles du jeu, du périmètre de ses acteurs, des contraintes qui les affectent. Risquerait-on d'ouvrir en même temps la porte à quelque posture antipolitique, de diaboliser élus et candidats? Ce serait confondre un diagnostic argumenté avec une prise de parti. Ce serait oublier l'éclairage paradoxal, très abouti chez Ben Cramer, qui rend intelligibles les talents éminents - et la variété de leurs combinatoires - que requiert un métier dont la visibilité ne semble plus avoir d'égale que l'impuissance à changer le monde ou le quotidien. En cela le New Journalism politique vient aussi rappeler que dresser le tableau clinique et critique d'un monde social peut receler le désir de pouvoir y réinvestir une illusio, une énergie créatrice. C'est enfin par tous ces traits que le meilleur de ce journalisme rejoint le meilleur des sciences sociales : dans l'ambition de démonter des causalités, de ne pas étouffer sous leur poids les singularités et la créativité des acteurs, dans la formulation d'une critique dont il soit possible de s'armer pour produire action et changement, non cynisme et ressentiment.

\section{Références}

Anonyme [KLein Joe], 1999, Primary Colours, New York, Random House.

Ben Cramer Richard, 1992, What It Takes. The Way to the White House, New York, Vintage.

Воотн Wayne, 1973, «Loathing and ignorance on the campaign trail 1972 », Columbia Journalism Review, vol.XII (4).

Boynton Robert, 2005, The New New Journalism, New York, Vintage.

Clayman Steven, Elliott Marc, Heritage John, BeCKett Megan, 2010, «A watershed in White House journalism. Explaining the post 1968 rise of aggressive presidential news », Political Communication, vol. XXVII (3), p. 229-247.

Collovald Annie, 2004, Le populisme du FN. Un dangereux contresens, Bellecombeen-Bauges, Le Croquant.

Crouch Colin, 2004, Post Democracy, Cambridge, Polity.

Crouse Timothy, 1973, The Boys in the Bus, New York, Random House.

Cullen Mike, 2012, Whiny Little Bitch. The Excuse-Filled Presidency of Barack Obama, Washington, Quite Right Books.

Didion Joan, 1968, Slouching Towards Bethlehem, New York, Farrar Straus and Giroux (trad. française : L’Amérique, Paris, Grasset, 2009). 
- 2001, Political Fictions, New York, Farrar, Straus and Giroux.

Freddoso David, 2011, Gangster Governement. Barack Obama and the New Washing-

ton Thugocracy, Washington DC, Regnery Publishing.

Gaxie Daniel, 1993, Le cens caché, Paris, Le Seuil.

Greenberg Meg, 2001, Washington, New York, Public Affairs.

HARR Jonathan, 1995, A Civil Action, New York, Vintage (trad. française : Préjudice, Paris, Pocket, 2003).

HaY Colin, 2007, Why We Hate Politics? Cambridge, Polity.

HeLLMAN John, 1981, Fables of Fact. The New Journalism as New Fiction, Urbana, University of Illinois Press.

Herr Michael, 1977, Dispatches, New York, Knopf (trad. française : Putain de mort, Paris, Le livre de poche, 1981).

Hersch Seymour, 1997, The Dark Side of Camelot, New York, Harper and Collins.

Hollowell John, 1977, Fact and Fiction. The New Journalism and the Non-Fiction Novel, Chapel Hill, The University of North Carolina Press.

JUNGER Sebastian, 2010, War, New York, Twelve (trad. française : Guerre, Paris, De Fallois, 2012).

KACIAF Nicolas, 2013, Les pages «Politique». Histoire du journalisme politique dans la presse française (1945-2006), Rennes, Presses universitaires de Rennes.

KLEIN Joe, 1997, Politics Lost. How American Politics Was Trivialised by People Who Think You Are Stupid, New York, Doubleday. Voir également AnonymE, supra.

LeBlanc Adrian Nicole, 2003, Random Family, New York, Anchor (trad. française : Les enfants du Bronx, Paris, L'Olivier, 2005).

LeWIS Michael, 1990, Liar's Poker, Philadelphia, Coronet Books.

- 1997, Trail Fever. Spin doctors, Rented Strangers, Thumb Wrestlers, Toe Suckers, Grizzly Bears and Other Creatures on the Road to the White House, New York, Alfred Knopf.

MCGINNISS Joe, 1969, The Selling of the President 1968, New York, Trident / Simon and Schuster.

MeYER Philip, Precision Journalism. A Reporter's Introduction to Social Science Methods, Bloomington, Indiana University Press.

NeVEu Erik, 2012a, Les Mots de la communication politique, Toulouse, Presses universitaire du Mirail.

- 2012b, "Nouveaux" journalismes d'enquête et sciences sociales. Penser emprunts, écarts et hybridations », Tracés, nº12, p. 225-243.

- 2013, "The primacy of persons? Why not, but a disenchanted one. A French sociologist visits Schabertpolis », dans J. Von Heyking, T. Heilke éd. (infra), p. 239-280.

Thompson Hunter S., 1966, Hell's Angels. The Strange and Terrible Saga of the Outlaw Motorcycle Gangs, New York, Random House (trad. française : Hell's Angels, Paris, UGE 10/18, 2004).

- 1973, Fear and Loathing. On the Campaign Trail' 72, New York, Grand Central.

- 2010, Generation of Swine. Tales of Shame and Degradation in the 80's, New York, Simon and Schuster.

Von Heyking John, Heilke Thomas, The Primacy of Persons in Politics, Empirism and Political Philosophy, Washington DC, The Catholic University of America Press. 
Weber Max, 1918, Le savant et le politique, Paris, UGE 10/18.

WhITE Theodore, 1961, The Making of the President 1960, New York, Atheneum Publishers.

- 1969, The Making of the President 1968, New York, Atheneum Publishers.

WoLfe Tom, 1965, The Kandy-Kolored Tangerine-Flake Streamline Baby, New York, Farrar, Straus and Giroux.

- 1968, The Electric Kool-Aod Acid Test, New York, Farrar, Straus and Giroux (trad. française : Acid Test, Paris, Le Seuil, 1996).

- 1970, Radical Chic \& Mau-Mauing the Flak Catchers, New York, Farrar, Straus and Giroux.

- 1973, The New Journalism, Basingstoke, Picador.

Woodward Bob, 1996, The Choice. How Bill Clinton Won, New York, Simon and Schuster.

Zavarzadeh Mas‘ud, 1976, The Mythopoetic Reality. The Postwar American Novel, Urbana, University of Illinois Press. 